\title{
Intensity-dependent bleaching relaxation in lead salt quantum dots
}

\author{
Vasili G. Savitski, Alexander M. Malyarevich, Mikhail I. Demchuk, and Konstantin V. Yumashev \\ International Laser Center, Building 17, \#65 F. Skaryna Ave., 220013 Minsk, Belarus \\ Helga Raaben and Alexander A. Zhilin \\ S. I. Vavilov State Optical Institute, St. Petersburg, Russia
}

Received July 16, 2004; revised manuscript received December 3, 2004; accepted March 11, 2005

\begin{abstract}
Bleaching relaxation in lead salt $(\mathrm{PbS})$ quantum dots ( $\mathrm{QDs}$ ) of various sizes and under different pump intensities has been studied. The observed bleaching relaxation features (particularly, shortening of the bleaching relaxation times with a decrease in the QD size and an increase of the pump-light intensity) are explained in the context of the proposed spectroscopic model. The model takes into account transitions of the excited-charge carriers both within the system of quantum-confined energy levels and the defect states of the QDs. The characteristic times of the direct electron-hole recombination, carriers trapping to the defect states, and their subsequent relaxation from these states as well as the cross sections of the ground-state and excited-state absorption of charge carriers can be evaluated from the experimental data for PbS QDs of different sizes and under different pumping conditions using this model. () 2005 Optical Society of America
\end{abstract}

OCIS codes: $160.2750,160.4760,300.6500,320.7130$.

\section{INTRODUCTION}

Semiconductor zero-dimensional structures (quantum dots, or QDs) have been intensively studied for the past 20 years, ${ }^{1-3}$ mainly because of their interesting physical nature-various effects caused by quantum confinement of charge carriers (electrons and-or holes) in structures of small sizes (comparable with exciton Bohr radius). Lead salt $(\mathrm{PbS})$ has a relatively large exciton $\mathrm{Bohr}$ radius $(18 \mathrm{~nm})$, and a strong quantum confinement is expected in such QDs in contrast with II-VI and I-VII semiconductors. On the other hand, enhanced optical nonlinearities within the range of the first excitonic resonance make possible the application of QDs as passive shutters for laser-pulse generation. A rather narrow bandgap in $\mathrm{PbS}$ provides a shifting of a spectral position of the first excitonic resonance from $1 \mu \mathrm{m}$ up to $3 \mu \mathrm{m}$ by increasing the QD size. A transient bleaching of the first excitonic absorption band in $\mathrm{PbS}-\mathrm{QD}$-doped glasses was exploited to $Q$ switch and mode lock a number of solid-state lasers emitting within the $1.06-1.55 \mu \mathrm{m}$ spectral region. ${ }^{4-11}$

It has been shown previously that the bleaching relaxation within the range of the first excitonic resonance for $\mathrm{PbS}$ QDs demonstrates double-exponential type, ${ }^{5,7,12-14}$ and the bleaching relaxation time depends on pump-light intensity. ${ }^{5,12,14}$ For PbS QDs of $5.4 \mathrm{~nm}$ in diameter the shortening of the fast relaxation time from 17 to $5 \mathrm{ps}$ with an increase in the $120-\mathrm{fs}$ pump fluence from 0.5 to $4 \mathrm{~mJ} / \mathrm{cm}^{2}$ was reported. ${ }^{12}$ The same tendency was registered for $\mathrm{PbS}$ QDs of $5.5 \mathrm{~nm}$ in diameter when the pump intensity was changed from 0.3 to $2.5 \mathrm{GW} / \mathrm{cm}^{2}$ (Ref. 14). However, no dependence on pump intensity was observed for QDs of $4.4 \mathrm{~nm}$ in diameter under picosecond pump fluences of $4-70 \mathrm{~mJ} / \mathrm{cm}^{2}$ (Ref. 13). It was shown ${ }^{7}$ that the fast bleaching relaxation time $\tau_{f}$ depends also on
$\mathrm{PbS}$ QDs size. A very strong dependence of the fast relaxation time on mean dots radius $\left(\tau_{f} \sim R^{9}\right)$ for PbS QDs of $4.7-5.8 \mathrm{~nm}$ in diameter dispersed in phosphate glass was reported. ${ }^{7}$

To describe the bleaching relaxation processes in $\mathrm{PbS}$ QDs of $5.5 \mathrm{~nm}$ in size, a four-level spectroscopic model was suggested. ${ }^{14}$ This model included excited-state absorption (ESA) between excitonic states and considered excitation relaxation by direct electron-hole recombination and recombination through defect states. This model was successfully used to simulate the performance of a 1.3- $\mu \mathrm{m} \mathrm{Nd}: \mathrm{YVO}_{4}$ laser passively $Q$ switched with $\mathrm{PbS}-$ QD-doped glass saturable absorber. ${ }^{15}$ However, the bleaching relaxation simulation within the frame of this model led to a conclusion that the characteristic time of the direct electron-hole recombination depends on pumplight intensity (i.e., the increasing of the light intensity from 0.3 to $2.5 \mathrm{GW} / \mathrm{cm}^{2}$ leads to the shortening of the characteristic time from 100 to $40 \mathrm{ps}),{ }^{14}$ which can be hardly explained.

As soon as saturable absorbers work in different lasers under various pump conditions, for the efficient application of $\mathrm{PbS}$ QDs as passive shutters it is necessary to know how their bleaching characteristics depend on excitation light parameters.

In the present work we report the detailed study of bleaching relaxation for $\mathrm{PbS} \mathrm{QDs}$ of various sizes $(4.2-6.3 \mathrm{~nm})$ under different excitation intensities and durations. In particular, the same PbS-QD-doped glass sample has been studied under picosecond and nanosecond pump-light excitation. It has been shown that $\mathrm{PbS}$ QDs demonstrate remarkably different nonsaturable (residual) absorption losses under the various pump-pulse durations. The observed features can be explained in the 

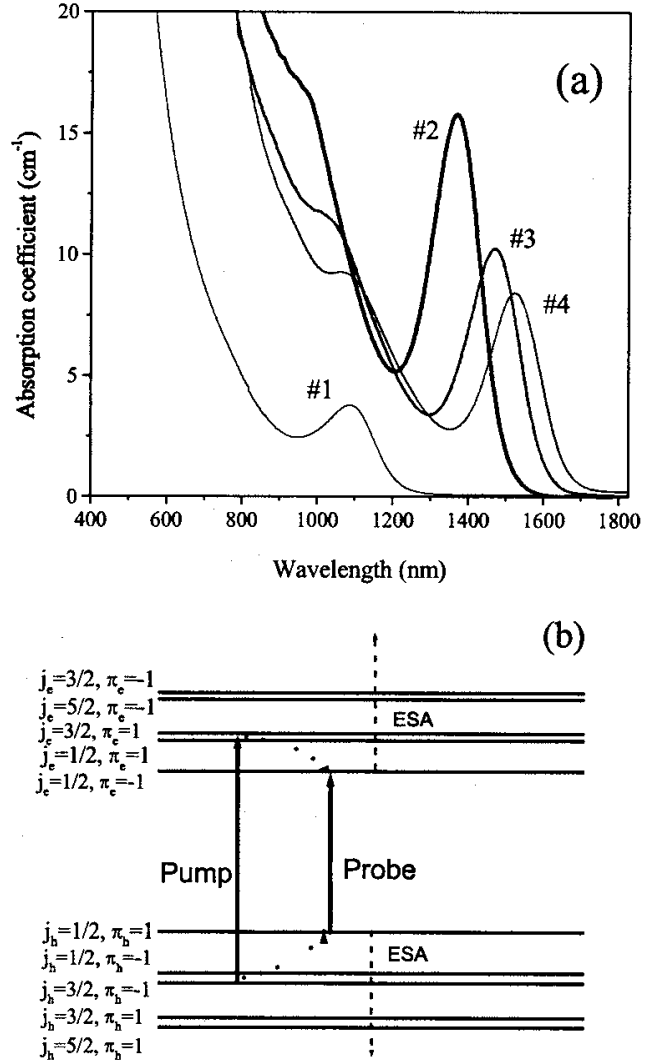

Fig. 1. (a) Absorption spectra of PbS-QD-doped silicate glasses with a dot average diameter of 4.2, 5.5, 6.0 and $6.3 \mathrm{~nm}$ (samples \#1-\#4, respectively). (b) Energy-level scheme of PbS QDs under the isotropic approximation. ${ }^{16}$

context of the modified spectroscopic model, which takes into account one more channel for ESA-within the system of the QDs defect states (in addition to the one suggested in Ref. 14, within the system of size-quantized levels). As a result, this model not only describes correctly the intensity-dependent transmission-absorption measurements but also allows the evaluation of the characteristic times of the direct electron-hole recombination, which does not depend any more on the pump-light intensity (in contrast to the model in Ref. 14) for PbS QDs of all sizes studied.

\section{EXPERIMENTAL DETAILS}

In the present work we have studied four PbS-QD-doped silicate glass samples. Their preparation was described earlier. ${ }^{4}$ These samples demonstrate the first excitonic absorption maximum at 1085 (sample \#1), 1370 (\#2), 1470 (\#3), and $1525 \mathrm{~nm}$ (\#4) [Fig. 1(a)], which corresponds to the mean dot diameters of $4.2,5.5,6.0$, and $6.3 \mathrm{~nm}$, respectively. The QD diameters have been determined from the dependence of the spectral position of the first excitonic peak on the $\mathrm{PbS}$ dot's diameter. ${ }^{16}$

The kinetics of bleaching relaxation was measured with a standard picosecond pump-probe technique described in detail in Ref. 17. A 15 ps pulse from the passively mode-locked $\mathrm{Nd}$ : $\mathrm{YAlO}_{3}$ laser $(\lambda=1.08 \mu \mathrm{m})$ was employed as a pump radiation. The pump intensity on the samples was varied from $\sim 0.04$ up to $\sim 6 \mathrm{GW} / \mathrm{cm}^{2}$ by neu- tral filters. The fundamental emission wavelength of the laser $(1.08 \mu \mathrm{m})$ and the second and the third Raman Stokes $(1.29$ and $1.52 \mu \mathrm{m})$ from the $\mathrm{KGd}\left(\mathrm{WO}_{4}\right)_{2}$ crystal, excited by a part of the pump pulse mentioned above, were used as a probe.

It should be noted that the $1.08 \mu \mathrm{m}$ pump excites a group of the higher-energy excitonic transitions in QDs for samples \#2, \#3, and \#4 [as seen in Fig. 1(b)]. Following pumping, the excited electrons and holes relax from the pumped energy levels to the lowest energy states [with $j_{e(h)}=1 / 2$; Fig. 1(b)] during $1-2$ ps. ${ }^{12,18}$ The characteristic time of this relaxation process is much shorter than the $15 \mathrm{ps}$ duration of the pump pulses, and it has not been resolved in our experiments. With the probe we register the kinetics of bleaching relaxation from the lowest energy excitonic state [Fig. 1(b)].

The absorption-saturation measurements in the nanosecond time scale have been performed at 1.06, 1.34, and $1.54 \mu \mathrm{m}$ with passively $Q$-switched Nd:YAG (pulse duration of $10 \mathrm{~ns}$ ), Nd: $\mathrm{YAlO}_{3}$ (50 ns), and Er:glass (65 ns) lasers, respectively. The pump-light intensity has been varied by moving the sample along the focused beam ( $z$-scan-like technique; see Ref. 19 for more details). The passively mode-locked $\mathrm{Nd}: \mathrm{YAlO}_{3}$ laser $(\lambda=1.08 \mu \mathrm{m})$ mentioned above has been applied for the absorptionsaturation measurements in the picosecond time scale. The transmittance of the 15 ps probe pulses at 1.08, 1.29, or $1.52 \mu \mathrm{m}$ has been measured after a $15 \mathrm{ps}$ time delay of the pump pulse at $1.08 \mu \mathrm{m}$ (i.e., measurements of the values of maximal optical density change $\triangle O D$ at the appro-

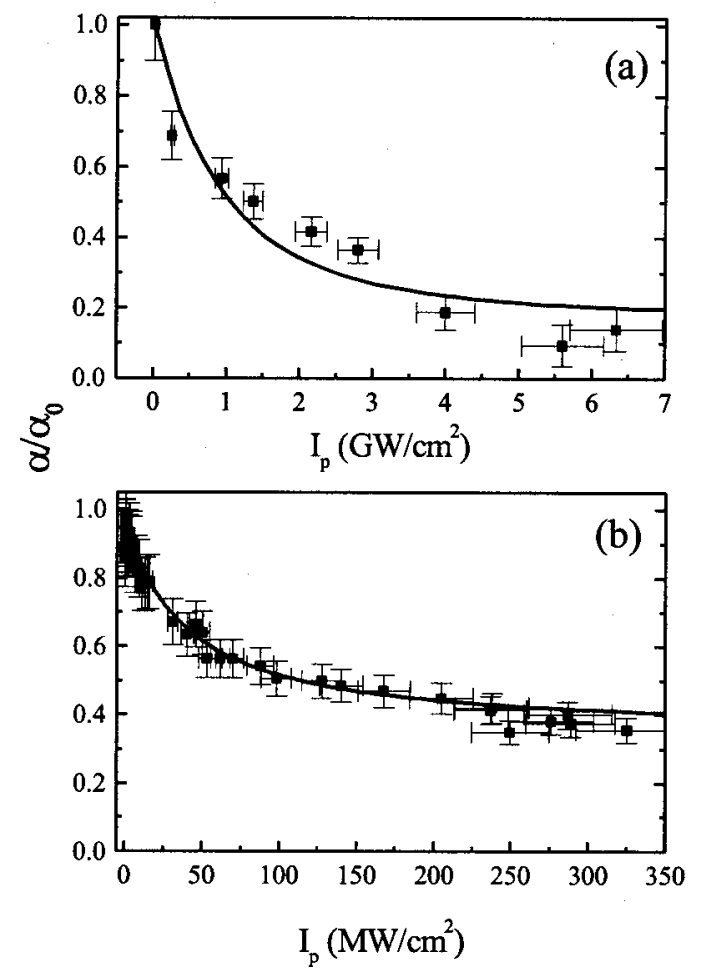

Fig. 2. Intensity-dependent relative absorption for the PbS-QDdoped glass sample \#3 (squares): (a) at $\lambda=1.525 \mu \mathrm{m}$ under the picosecond pump; (b) at $\lambda=1.54 \mu \mathrm{m}$ under the nanosecond excitation. Solid curves present the results of the best fit within Eqs. (2) and (5), respectively. 

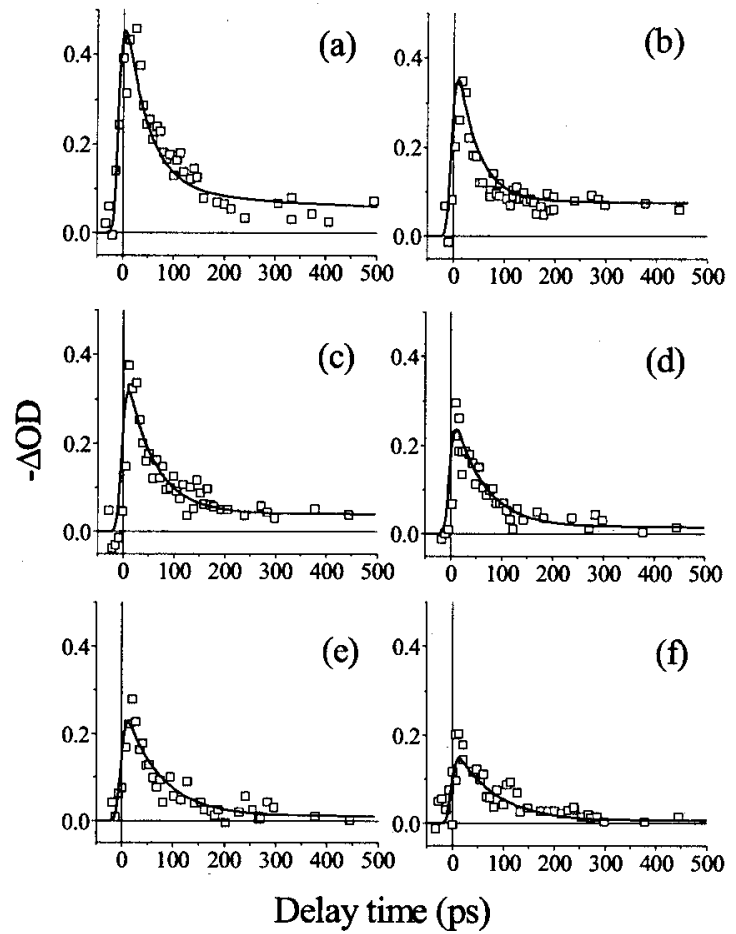

Fig. 3. Kinetics of bleaching relaxation for the sample \#3 at $1.52 \mu \mathrm{m}$ under the pump intensities of $5.6,2.8,2.2,1.4,0.9$, and $0.25 \mathrm{GW} / \mathrm{cm}^{2}$ (a)-(f), respectively. Solid curves present the results of the modeling using the rate equations [Eq. (2)] and the parameters of the spectroscopic model from Table 1.

priate pump intensities have been performed). Neutral filters have been used to vary the pump intensity.

The damage threshold of the samples has been estimated to be $\sim 10 \mathrm{~J} / \mathrm{cm}^{2}$ (for the nanosecond pulse duration) and $\sim 15 \mathrm{GW} / \mathrm{cm}^{2}$ (for the picosecond pulse duration).

\section{EXPERIMENTAL RESULTS}

\section{A. Absorption Saturation Data}

Figure 2 presents the intensity-dependent relative absorption of $\mathrm{PbS}-\mathrm{QD}$-doped glass (sample \#3) registered under picosecond [Fig. 2(a)] and nanosecond [Fig. 2(b)] excitation. The picosecond measurements have been done at the wavelength of $1.52 \mu \mathrm{m}$ and the nanosecond ones at $1.54 \mu \mathrm{m}$.

One can see that a powerful picosecond pump leads to lower residual absorption $\alpha_{\mathrm{ns}} / \alpha_{0}=0.09\left[\alpha_{0}\right.$, small-signal absorption coefficient; Fig. 2(a)]. On the other hand, the nanosecond pump [Fig. 2(b)] leaves much higher residual losses of $\alpha_{\mathrm{ns}} / \alpha_{0}=0.37$. The ratios $\alpha_{\mathrm{ns}} / \alpha_{0}$ have been estimated from the experimental data [Figs. 2(a) and 2(b)] at maximal pump intensities available. It should be mentioned that this difference in the $\alpha_{\mathrm{ns}} / \alpha_{0}$ value is consistent with the earlier registered results. The value of $\alpha_{\mathrm{ns}}$ $=0.03 \mathrm{~cm}^{-1} \quad\left(\alpha_{\mathrm{ns}} / \alpha_{0}=0.02\right)$ was achieved under $33 \mathrm{ps}$ pump pulse. ${ }^{6}$ For the nanosecond excitation, values of $\alpha_{\text {ns }} / \alpha_{0} \approx 0.2-0.4$ were obtained. ${ }^{4,9}$ This pronounced difference in the residual absorption registered for the "short" and "long" pump shows that these losses cannot be associated with nonsaturable absorption owing to the scattering processes and the imperfection of the glass sample.
One possible explanation is the presence of two different channels for ESA in PbS QDs, which manifest themselves mainly under the nanosecond or picosecond pump. These channels are: ESA within the system of $\mathrm{PbS} \mathrm{QD}$ s excitonic states from the lowest excitonic level to the higher (with $j_{e(h)}>5 / 2$ ) electron and-or hole states, as proposed in Ref. 14 (this ESA is revealed by the picosecond excitation); and ESA within the system of energy levels related to the various in nature defect states (including dot-surface states), as observed under the "long" nanosecond pump.

\section{B. Transient Bleaching Data}

Figure 3 presents the results of the bleaching relaxation measurements under different pump intensities (from 0.25 to $5.6 \mathrm{GW} / \mathrm{cm}^{2}$ ) for the $\mathrm{PbS} \mathrm{QDs}$ of $6.0 \mathrm{~nm}$ in size (sample \#3). The kinetics of the bleaching relaxation is characterized with two components-fast and slow-and can be described by the equation

$$
-\Delta O D=A_{f} \exp \left(-t / \tau_{f}\right)+A_{s} \exp \left(-t / \tau_{s}\right),
$$

where $A_{f}, A_{s}, \tau_{f}$, and $\tau_{s}$ are the amplitudes and the relaxation times of the fast and slow bleaching components. One can see that within the time interval of $200-500$ ps the $\triangle O D$ signal decreases to a plateau $\Delta O D_{p l}$ for all pump intensities. The time delay in our experiments is not long enough to correctly measure $\tau_{s}$ values, so we estimate these as lying in at least a nanosecond time scale, and we also evaluate $A_{s}$ amplitudes as the values of $\Delta O D_{p l}$ at the appropriate pump intensities. The fast component is attributed to the direct electron-hole recombination or relaxation through the shallow defect states. To explain the emergence of the second (slow) bleaching relaxation component, we postulated the carriers' (electrons and-or holes) trapping to the deep defect (surface) states with rather long (nanosecond) live time. ${ }^{13}$ The change in excitation intensity leads to the variation of the values of $\tau_{f}$ and $A_{f} / A_{s}$. The appropriate data are collected in Table 1. The tendency is the shortening of the time of the fast component $\tau_{f}$ and the decreasing of the $A_{f} / A_{s}$ value with the increasing of the pump intensity $I_{p}$ (Fig. 4). The same behavior was registered for $\mathrm{PbS} Q \mathrm{QDs}$ of $\approx 5.5 \mathrm{~nm}$ in diameter. $^{12,14}$ The time-delay interval in our experiments has not been long enough to register definitely the change of the $\tau_{s}$ value with the change of $I_{p}$.

\section{DISCUSSION}

Relaxation processes in $\mathrm{PbS}$ QDs can be described within the frame of the model presented in Fig. 5. This model includes level $\langle 0\rangle$, a ground state, and two groups of the excited state energy levels: $\langle 1\rangle$, the first excited state; $\langle 2\rangle$, a higher excited state within the system of quantum confined states in the $\mathrm{QD}$; and $\mathrm{DS}$, the defect states (we do not detail the nature of these defects; they are most probably due to the dot-surface interface). If we compare this model with the energy structure of $\mathrm{PbS}$ QDs under the isotropic approximation [Fig. 1(b)], ${ }^{16}$ the transition $\langle 0\rangle$ $\rightarrow\langle 1\rangle$ corresponds to the electrons and holes excitation to the levels $\left|j_{e}=1 / 2, \pi_{e}=-1\right\rangle$, and $\left|j_{h}=1 / 2, \pi_{h}=1\right\rangle$, respectively. The higher excited state $\langle 2\rangle$ corresponds to the electron (hole) state with the quantum number $j>5 / 2$ [not 


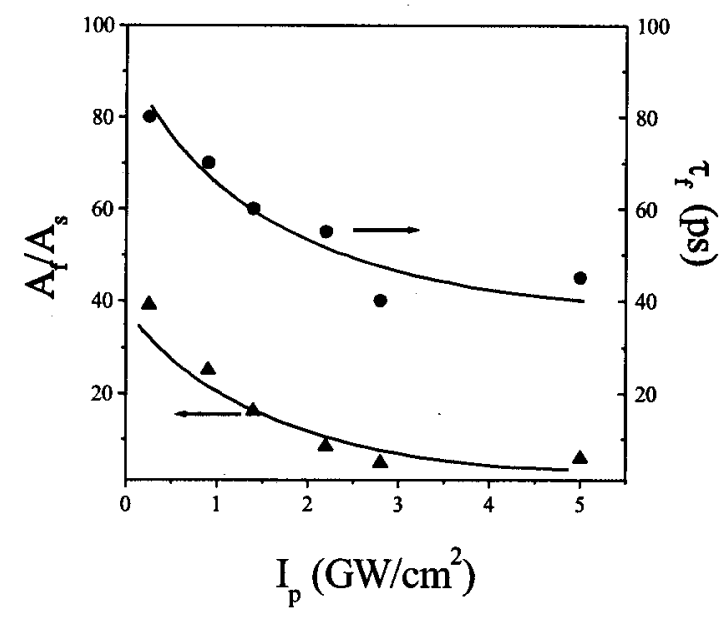

Fig. 4. Dependencies of the fast relaxation time $\tau_{f}$ and the ratio of fast to slow component amplitudes $A_{f} / A_{s}$ on the pump intensity. Solid curves are the guides for eyes.

shown in Fig. 1(b)]. The depopulation of the level $\langle 1\rangle$ takes place during tens of picoseconds (time $\tau_{f}$ registered in pump-probe experiments), so the ESA between levels $\langle 1\rangle$ and $\langle 2\rangle$ is observed only within this time interval. During this period a part of electrons and holes recombine, and other excited carriers are trapped to the defect states. After this trapping, the ESA within the channel of the defect states raises. This ESA is observed for several nanoseconds (time $\tau_{s}$ ). So, the intensity-dependent transmission measurement in the nanosecond time scale reveals this long-lived ESA. It is possible to observe separately these two ESA processes in the experiment. Using "short" (several units or several tens of picoseconds) pump pulses, one registers only ESA within the channel $\langle 1\rangle \rightarrow\langle 2\rangle$, because there are no carriers in the DS (or their amount is negligible). When the pump intensity of the picosecond pulse is very high $\left(I_{p} \rightarrow \infty\right)$, the maximal bleaching signal $\Delta O D_{\max }\left(I_{p} \rightarrow \infty\right)$ is determined by the value of $\sigma_{1} / \sigma_{0}\left(\sigma_{0}\right.$ and $\sigma_{1}$ are the ground-state and the excited-state absorption cross sections of the transitions $\langle 0\rangle \rightarrow\langle 1\rangle$ and $\langle 1\rangle$ $\rightarrow\langle 2\rangle$, respectively). At "long" (nanosecond and longer) pumping, the DS are mostly populated (owing to the picosecond relaxation time $\tau_{f}$ the population of the level $\langle 1\rangle$ is negligible), the ESA due to the trapped carriers is only observed. So, the value of $\sigma_{2} / \sigma_{0}$ determines the residual absorption [or saturated transmittance $T_{\text {sat }}\left(I_{p} \rightarrow \infty\right)$ ] under the high intensive nanosecond excitation $\left(\sigma_{2}\right.$ is the excited-state absorption cross-section due to the trapped carriers),

As a result, the processes of absorption and excitedstate relaxation can be described in accordance with the energy-level scheme in Fig. 5 using the rate equations:

$$
\begin{aligned}
& \mathrm{d} N_{0} / \mathrm{d} t=-I_{p}(t) \sigma_{p} N_{0}(t)+N_{1}(t) / \tau_{1}+N_{3}(t) / \tau_{3}, \\
& \mathrm{~d} N_{1} / \mathrm{d} t=I_{p}(t) \sigma_{p} N_{0}(t)-N_{1}(t) / \tau_{1}-N_{1}(t) / \tau_{13}, \\
& \mathrm{~d} N_{3} / \mathrm{d} t=N_{1}(t) / \tau_{13}-N_{3}(t) / \tau_{3},
\end{aligned}
$$

$$
\begin{aligned}
\Delta O D\left(t_{d}\right) \propto & \int_{-\infty}^{+\infty} \exp \left[-2\left(t-t_{d}\right) / \Delta t\right]^{2}\left\{-\left[2 N_{1}(t)\right.\right. \\
& \left.\left.+N_{3}(t)\right]+\frac{\sigma_{1}}{\sigma_{0}} N_{1}(t)+\frac{\sigma_{2}}{\sigma_{0}} N_{3}(t)\right\} \mathrm{d} t \\
I_{p}(t)= & I_{p 0} \exp (-2 t / \Delta t)^{2}
\end{aligned}
$$

where $N_{0}, N_{1}$, and $N_{3}$ are the populations of the levels $\langle 0\rangle$, $\langle 1\rangle$ and the DS on which the excited carriers are accumulated, respectively; $\sigma_{p}$ is the ground-state absorption cross-section at the wavelength of the pump; $\Delta t$ is the pump-pulse width (15 ps). The population of the level $\langle 2\rangle$, $N_{2}$ is negligible because of the short relaxation time $\tau_{2}$ $(1-2 \mathrm{ps})^{12}$ in comparison with the pump-pulse width $\Delta t$. Analogously we neglect the population of the appropriate (in energy) higher defect level expecting its short relaxation time.

The experimental data provide five values: $\Delta O D_{\max }\left(I_{p}\right.$ $\rightarrow \infty), T_{\text {sat }}\left(I_{p} \rightarrow \infty\right), A_{f} / A_{s}, \tau_{f}$ and $\tau_{s}$, which refer to the physical parameters from the spectroscopic model (Fig. 5) as following:

$$
\begin{aligned}
\gamma_{1} & =1-\frac{\sigma_{1}}{2 \sigma_{0}} \\
\gamma_{2} & =\left[\frac{\sigma_{1}}{\sigma_{0}} \frac{\tau}{\tau_{3}}+\frac{\sigma_{2}}{\sigma_{0}}\left(1-\frac{\tau}{\sigma_{1}}\right)\right] /\left[1-\frac{\tau}{\tau_{1}}+2 \frac{\tau}{\tau_{3}}\right], \\
a & =\left[\left(1-\frac{\tau}{\tau_{3}}\right)\left(2-\frac{\sigma_{1}}{\sigma_{0}}\right) /\left(1-\frac{\tau}{\tau_{1}}\right)\left(1-\frac{\sigma_{2}}{\sigma_{0}}\right)\right]-1, \\
\tau_{s} & =\tau_{3}, \\
\tau_{f} & =\frac{\tau_{1} \tau_{13}}{\tau_{1}+\tau_{13}}
\end{aligned}
$$

where $\gamma_{1}=\left|\Delta O D_{\max }\left(I_{p} \rightarrow \infty\right)\right| / D_{0}, \quad \gamma_{2}=\ln T_{\text {sat }}\left(I_{p} \rightarrow \infty\right) / \ln T_{0}$, $a=A_{f} / A_{s}, \tau=\left(\tau_{1} \tau_{13}\right) /\left(\tau_{1}+\tau_{13}\right) \equiv \tau_{f}$, and $D_{0}$ is the initial optical density of the sample. With the experimental data it is

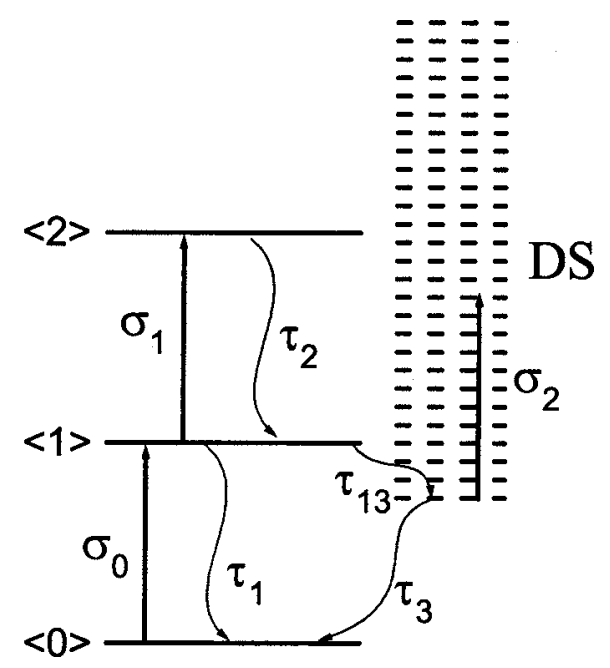

Fig. 5. Spectroscopic model for the description of the bleaching relaxation processes in $\mathrm{PbS}$ QDs. 
Table 1. Experimental and Calculated Data on Bleaching Relaxation in PbS QDs ${ }^{a}$

\begin{tabular}{|c|c|c|c|c|c|c|c|c|c|c|}
\hline \multirow[b]{2}{*}{$\begin{array}{c}D \\
(\mathrm{~nm})\end{array}$} & \multicolumn{6}{|c|}{ Experiment } & \multicolumn{4}{|c|}{ Model } \\
\hline & $\gamma_{1}$ & $\gamma_{2}$ & $\begin{array}{c}I_{p} \\
\left(\mathrm{GW} / \mathrm{cm}^{2}\right)\end{array}$ & $\begin{array}{c}\tau_{f} \\
(\mathrm{ps})\end{array}$ & $\begin{array}{c}\tau_{s} \\
(\mathrm{~ns})\end{array}$ & $A_{f} / A_{s}$ & $\sigma_{1} / \sigma_{0}$ & $\sigma_{2} / \sigma_{0}$ & $\begin{array}{c}\tau_{1} \\
(\mathrm{ps})\end{array}$ & $\begin{array}{c}\tau_{12} \\
(\mathrm{ps})\end{array}$ \\
\hline 4.2 & 0.65 & 0.43 & 0.90 & $20 \pm 2$ & $0.1 \pm 0.02$ & 2.0 & 0.7 & 0.49 & 63 & 30 \\
\hline \multirow[t]{3}{*}{5.5} & 0.55 & 0.45 & 0.30 & $28 \pm 3$ & $>1$ & 2.3 & 0.9 & 0.45 & 71 & 46 \\
\hline & & & 0.90 & $25 \pm 3$ & & 1.9 & & 0.45 & 77 & 37 \\
\hline & & & 2.50 & $20 \pm 3$ & & 1.8 & & 0.45 & 69 & 28 \\
\hline \multirow[t]{6}{*}{6.0} & 0.95 & 0.35 & 0.25 & $80 \pm 6$ & $>1$ & 39.0 & 0.1 & 0.47 & 88 & 900 \\
\hline & & & 0.90 & $70 \pm 7$ & & 25.0 & & 0.45 & 80 & 580 \\
\hline & & & 1.40 & $60 \pm 5$ & & 16.0 & & 0.41 & 73 & 330 \\
\hline & & & 2.20 & $55 \pm 5$ & & 8.3 & & 0.39 & 82 & 164 \\
\hline & & & 2.80 & $40 \pm 4$ & & 4.7 & & 0.38 & 87 & 74 \\
\hline & & & 5.60 & $45 \pm 4$ & & 5.7 & & 0.43 & 86 & 94 \\
\hline 6.3 & 0.40 & 0.40 & 6.00 & $50 \pm 4$ & $>1$ & 2.5 & 1.2 & 0.36 & 86 & 120 \\
\hline
\end{tabular}

${ }^{a} D$ is the QD diameter; $I_{p}$ is the pump intensity.

possible to define the spectroscopic parameters of the model from the following equations:

$\frac{\sigma_{1}}{\sigma_{0}}=2\left(1-\gamma_{1}\right)$

$\frac{\sigma_{2}}{\sigma_{0}}=\frac{\left(\tau_{f} / \tau_{s}\right)\left[\left(1-\gamma_{2}\right)\left(1+a-\gamma_{1}\right)-a \gamma_{1}\right]-\gamma_{1} \gamma_{2}}{\left(\tau_{f} / \tau_{s}\right)\left[\left(1-\gamma_{2}\right)(1+a)-a \gamma_{1}\right]-\gamma_{1}}$,

$\tau_{1}=\tau_{f} /\left[\left(1+2 \frac{\tau_{f}}{\tau_{s}}\right)-\frac{2 \gamma_{1}}{(1+a)\left(1-\gamma_{2}\right)}\left(1+a \frac{\tau_{f}}{\tau_{s}}\right)\right]$,

$\tau_{13}=\frac{\tau_{1} \tau_{f}}{\tau_{1}-\tau_{f}}$

$\tau_{3}=\tau_{s}$.

So, having carried out three types of the experimentsthe intensity-dependent absorption measurements under the "short" (picosecond) and "long" (e.g., nanosecond) pump, and the bleaching relaxation measurements using the picosecond pump-probe technique-one can evaluate the spectroscopic parameters (the characteristic times of relaxation, $\tau_{1}, \tau_{13}$, and $\tau_{3}$; and the ratios of the excitedstate to the ground-state absorption cross sections, $\sigma_{1} / \sigma_{0}$ and $\sigma_{2} / \sigma_{0}$ ) that describe the excitation and relaxation processes in $\mathrm{PbS}$ QDs.

It is possible with experimental data to calculate the spectroscopic parameters of the model based on Eqs. (4) and then to simulate numerically the relaxation processes in QDs within the frame of the rate equations [Eqs. (2)]. The intensity-dependent bleaching measurements under the picosecond excitation provide the maximal $\triangle O D$ value for the sample $\# 3$ as $\left|\Delta O D_{\max }\left(I_{p} \rightarrow \infty\right)\right|=0.52$ at $I_{p}$ $=5.6 \mathrm{GW} / \mathrm{cm}^{2}$ [see Fig. 2(a)]; as soon as the initial optical density $D_{0}=0.55$, so the value of $\gamma_{1}$ equals to 0.95 . The experiment for the nanosecond intensity-dependent transmission measurements leads to $T_{\text {sat }}\left(I_{p} \rightarrow \infty\right)=0.64$ at $I_{p}$ $=240 \mathrm{MW} / \mathrm{cm}^{2}$, which gives the value of $\gamma_{2}=\ln T_{\text {sat }}\left(I_{p}\right.$ $\rightarrow \infty) / \ln T_{0}=0.35 \quad\left(T_{0}\right.$ is a small-signal transmittance). These parameters, $\gamma_{1}$ and $\gamma_{2}$, are used to calculate the characteristics of the spectroscopic model at all pump intensities. The calculated parameters $\tau_{1}, \tau_{13}, \tau_{3}, \sigma_{1} / \sigma_{0}$ and $\sigma_{2} / \sigma_{0}$ are summarized in Table 1 . The results of the simulations of the bleaching relaxation processes in $\mathrm{PbS} Q \mathrm{QDs}$ (sample \#3) within the frame of the rate equations [Eqs. (2)] at different pump intensities are presented in Fig. 3 with solid curves. One can see that agreement between the calculated relaxation curve and the experimental data is quite good for all pump intensities.

The intensity-dependent transmittance under the "long" pump can be modeled by use of a simpler way than with the Eqs. (2). If the pump-pulse duration is much longer than the relaxation time $\tau_{3}$, the Eqs. (2) can be transformed to the analytical expression describing the intensity-dependent transmittance $T$ :

$$
\begin{aligned}
\ln \left(\frac{T}{T_{0}}\right)= & \left(\frac{\tau_{3}+2 \tau_{13}}{\tau_{3} \frac{\sigma_{2}}{\sigma_{0}}+\tau_{13} \frac{\sigma_{1}}{\sigma_{0}}}-1\right) \\
& \times \ln \left(\frac{1+I \tau_{1} \sigma_{1}\left(\frac{\sigma_{2}}{\sigma_{1}} \tau_{3}+\tau_{13}\right) /\left(\tau_{1}+\tau_{13}\right)}{1+I T \tau_{1} \sigma_{1}\left(\frac{\sigma_{2}}{\sigma_{1}} \tau_{3}+\tau_{13}\right) /\left(\tau_{1}+\tau_{13}\right)}\right)
\end{aligned}
$$

The modeled curve is presented in Fig. 2(b) with the solid curve showing good agreement with the experimental data.

The values of the parameters of the model obtained from the fitting of the experimental data at different intensities are presented in Fig. 6. One can see that the $\sigma_{2} / \sigma_{0}$ value (for the sample \#3) is changed between 0.38 and 0.47 [Fig. 6(a), solid squares; and Table 1]. This occurs, in our opinion, because the $\sigma_{2} / \sigma_{0}$ value depends on $\tau_{f}$ and $a=A_{f} / A_{s}$, which vary with the pump intensity. However, taking into account the experimental uncertainty of the picosecond pump-probe measurements ( $\approx 10 \%$ ) it is possible to conclude that $\sigma_{2} / \sigma_{0}$ has the same average value of $\sigma_{2} / \sigma_{0} \approx 0.43$ for all pump intensities $I_{p}$. On the other hand, it is reasonable that the ratio of the 

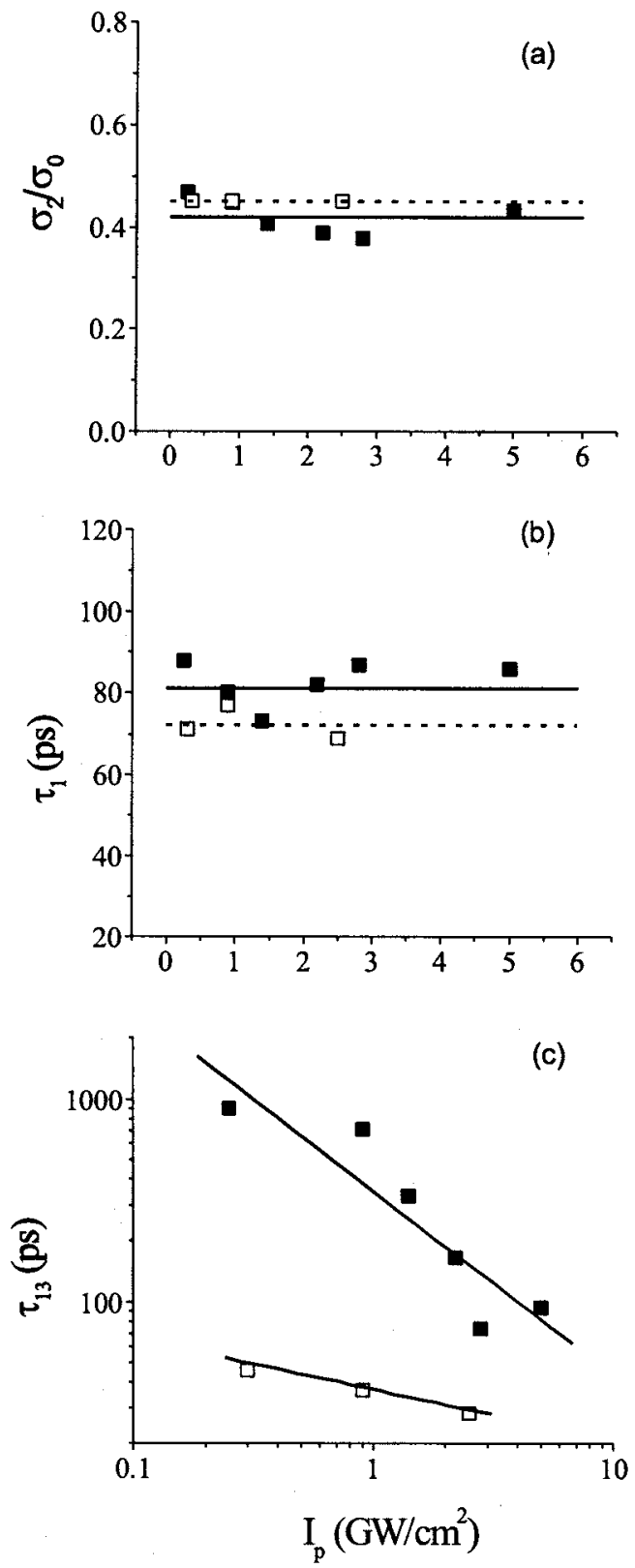

Fig. 6. Dependence of the parameters of the model (a) $\sigma_{2} / \sigma_{0}$, (b) $\tau_{1}$, and (c) $\tau_{13}$ on the pump intensity for PbS QDs of $6.0 \mathrm{~nm}$ (solid squares) and $5.5 \mathrm{~nm}$ (open squares) in size.

absorption cross sections does not vary with the pump intensity. The same type of dependence is observed for the time constant $\tau_{1}$; it is changed from 73 to $88 \mathrm{ps}$ for the sample \#3 [see Fig. 6(b), solid squares; and Table 1]. Analogously, one can evaluate the constant value of $\tau_{1}$ $\approx 80 \mathrm{ps}$ for all pump intensities. The parameter $\tau_{1}$ is the characteristic time of the direct electron-hole recombination, so obviously this constant has not to vary with the pump intensity. (It should be mentioned that the modeling based on the energy-level scheme suggested in Ref. 14 leads to the shortening of time constant $\tau_{1}$ with the increasing of the pump intensity, which could barely be explained.) In contrast, the time constant $\tau_{13}$ demonstrates the remarkable shortening with the increasing of the pump light intensity [Fig. 6(c), solid squares; and Table
1]. Constant $\tau_{13}$ characterizes the probability of the carriers trapping to the DS. The physical reason for the increased rate of this trapping process is not evident from our experiments; however, this may illustrate the strengthening of the dot-matrix interaction in a higherintensive electric field.

One more comment could be added, taking into account the possible usage of $\mathrm{PbS}-\mathrm{QDs}$-doped glasses as laser saturable absorbers. For such applications a low value of $\alpha_{\mathrm{ns}} / \alpha_{0}$ is preferable. For short (picosecond) pulses lowenough nonsaturable absorption can be achieved $\left[\alpha_{\mathrm{ns}} / \alpha_{0}\right.$ $<0.1$; see Fig. 2(a)]. However, longer excitation leads to the remarkable residual losses $\left(\alpha_{\mathrm{ns}} / \alpha_{0}>0.3\right)$, which could govern the pulse dynamics in $Q$-switched lasers. These losses are associated with the excited-state absorption within the system of the defect states in QDs, and the bleaching contrast may be probably improved by optimizing the $\mathrm{PbS}-\mathrm{QDs}$-doped glass preparation procedure.

\section{QUANTUM-DOT SIZE-DEPENDENT BLEACHING RELAXATION}

As shown earlier the bleaching relaxation characteristics for PbS QDs depend on dot size. ${ }^{7}$ Here we analyze the bleaching relaxation data for QDs of different sizes (samples \#1, \#3, and \#4) obtained in this research and earlier in Ref. 14 (sample \#2) using the rate equations [Eqs. (2)], as described above. The appropriate experimental data and the results of calculations and modeling are collected in Table 1 and for QDs of $5.5 \mathrm{~nm}$ in diameter are presented in Fig. 6 with open squares. The parameters of the model $\tau_{1}$ and $\tau_{13}$ for different sizes of QDs are compared in Fig. 7.

The value of the characteristic time $\tau_{1}$ is found to shorten from $85 \mathrm{ps}$ down to $60 \mathrm{ps}$ with the dot diameter decreasing from $6.3 \mathrm{~nm}$ down to $4.2 \mathrm{~nm}$ (Fig. 7, open squares). This shortening may be explained as a consequence of stronger interaction of the electron and hole wave functions with the matrix surrounding the smaller dots, which is a result of the extremely high carrier confinement in $\mathrm{PbS} \mathrm{QDs}$ of such sizes. The value of the characteristic time $\tau_{13}$ also becomes shorter for the smaller dots if one compares the values for the same pump intensity (e.g., $I_{p}=0.9 \mathrm{GW} / \mathrm{cm}^{2}$ in Fig. 7 , solid squares). So the trapping to the defect states occurs faster for QDs of a smaller size.

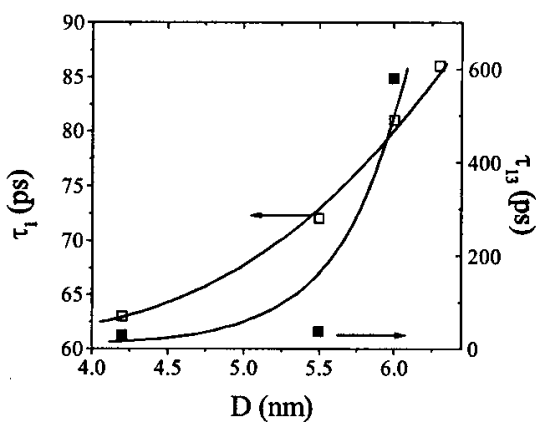

Fig. 7. Parameters of the model $\tau_{1}$ (open squares) and $\tau_{13}$ (solid squares) for PbS-QD-doped glass samples \#1-\#4 (see the text for details). Solid curves are the guides for eyes. 


\section{CONCLUSION}

The bleaching relaxation in $\mathrm{PbS}$ quantum dots for the different pump intensities $\left(0.25-6.0 \mathrm{GW} / \mathrm{cm}^{2}\right)$, pump-pulse durations (picosecond and nanosecond) and quantum dots diameters $(4.2-6.3 \mathrm{~nm})$ has been studied. It has been shown that the durable excitation (nanosecond) leads to a much higher residual absorption in $\mathrm{PbS}$ QDs then the short (picosecond) one ( $\alpha_{\mathrm{ns}} / \alpha_{0} \approx 0.4$ and 0.1 , respectively). For the QDs of $6 \mathrm{~nm}$ in size an increase in the pump intensity from 0.25 to $5.6 \mathrm{GW} / \mathrm{cm}^{2}$ (in $\approx 20$ times) causes the shortening of the fast relaxation characteristic time $\tau_{f}$ from 80 to $45 \mathrm{ps}$ and a decrease in the ratio of the fast component amplitude to the slow one $A_{f} / A_{s}$ in $\sim 1$ order (from 40 to 5 ). The observed features of the intensitydependent bleaching relaxation in $\mathrm{PbS}$ QDs are explained in the context of the proposed spectroscopic model. The parameters of the model (the characteristic times of the direct electron-hole recombination, excited carriers trapping to the defect states and their relaxation from these states to the ground state, and the ratios of excited-state to ground-state absorption cross-sections) can be evaluated from the experimental data. The analysis shows that the probability of carriers trapping to the defect states is enhanced with an increase of the pump intensity; for QDs of $6 \mathrm{~nm}$ in size the corresponding characteristic time shortens from $900 \mathrm{ps}$ down to $90 \mathrm{ps}$ with the pump intensity increasing from $0.25 \mathrm{GW} / \mathrm{cm}^{2}$ up to $5.6 \mathrm{GW} / \mathrm{cm}^{2}$. Also, this characteristic time becomes shorter for the smaller dots (with decreasing of the dot size from $6.0 \mathrm{~nm}$ to $4.2 \mathrm{~nm}$, the time constant shortens from $700 \mathrm{ps}$ to $30 \mathrm{ps}$ ) as taken at the fixed pump intensity.

V. G. Savitski is the corresponding author and can be reached by e-mail at savitskivass@rambler.ru or by fax at +375172326286.

\section{REFERENCES}

1. A. D. Yoffe, "Semiconductor quantum dots and related systems: electronic, optical, luminescence and related properties of low-dimensional systems," Adv. Phys. 50, 1-208 (2001).

2. S. V. Gaponenko, Optical Properties of Semiconductor Nanocrystals (Cambridge U. Press, 1998).

3. U. Woggon, Optical Properties of Semiconductor Quantum Dots (Springer, 1997).

4. A. M. Malyarevich, V. G. Savitski, P. V. Prokoshin, N. N. Posnov, K. V. Yumashev, E. Raaben, and A. A. Zhilin, "Glass doped with $\mathrm{PbS}$ quantum dots as a saturable absorber for 1- $\mu \mathrm{m}$ neodymium lasers," J. Opt. Soc. Am. B 19, 28-32 (2002).

5. A. Dementjev, V. Gulbinas, L. Valkunas, I. Motchalov, H. Raaben, and A. Michailovas, "Mode-locking of neodymium lasers by glasses doped with $\mathrm{PbS}$ nanocrystals," Appl. Phys. B 77, 595-599 (2003).

6. P. T. Guerreiro, S. Ten, N. F. Borrelli, J. Butty, G. E. Jabbour, and N. Peyghambarian, "PbS quantum-dot doped glasses as saturable absorbers for mode-locking of a Cr:forsterite laser,” Appl. Phys. Lett. 71, 1595-1597 (1997).

7. V. G. Savitski, N. N. Posnov, P. V. Prokoshin, A. M. Malyarevich, K. V. Yumashev, M. I. Demchuk, and A. A. Lipovskii, "PbS-doped phosphate glasses saturable absorbers for 1.3- $\mu \mathrm{m}$ neodymium lasers," Appl. Phys. B 75, 841-846 (2002).

8. V. G. Savitski, A. M. Malyarevich, K. V. Yumashev, B. D. Sinclair, A. A. Lipovskii, "Diode-pumped $\mathrm{Nd}: \mathrm{YVO}_{4}$ and $\mathrm{Nd}: \operatorname{KGd}\left(\mathrm{WO}_{4}\right)_{2} 1.3 \mu \mathrm{m}$ lasers passively $Q$-switched with PbS-doped glass," Appl. Phys. B 76, 253-256 (2003).

9. A. M. Malyarevich, I. A. Denisov, V. G. Savitsky, K. V. Yumashev, and A. A. Lipovskii, "Glass doped with PbS quantum dots as passive $Q$-switch for $1.54 \mu \mathrm{m}$ laser," Appl. Opt. 39, 4345-4347 (2000).

10. J. F. Philipps, T. Töpfer, H. Ebendorff-Heidepriem, D. Ehrt, R. Sauerbrey, and N. F. Borrelli, "Diode-pumped erbiumytterbium-glass laser passively $Q$-switched with a $\mathrm{PbS}$ semiconductor quantum-dot doped glass," Appl. Phys. B 72, 175-178 (2001).

11. A. A. Lagatsky, C. G. Leburn, C. T. A. Brown, W. Sibbett, A. M. Malyarevich, V. G. Savitski, K. V. Yumashev, E. L. Raaben, and A. A. Zhilin, "Passive mode locking of a $\mathrm{Cr}^{4+}$ :YAG laser by $\mathrm{PbS}$ quantum-dot-doped glass saturable absorber," Opt. Commun. 241, 449-454 (2004).

12. K. Wundke, S. Putting, J. Auxier, A. Schulzgen, N Peyghambarian, and N. F. Borrelli, "PbS quantum-dotdoped glasses for ultrashort-pulse generation," Appl. Phys. Lett. 76, 10-12 (2000).

13. G. Tamulaitis, V. Gulbinas, G. Kodis, A. Dementjev, L. Valkunas, I. Motchalov, and H. Raaben, "Optical nonlinearities of glass doped with $\mathrm{PbS}$ nanocrystals," $\mathrm{J}$. Appl. Phys. 88, 178-182 (2000).

14. V. G. Savitski, N. N. Posnov, A. M. Malyarevich, K. V. Yumashev, E. Raaben, and A. A. Zhilin, "Relaxation of bleaching in lead sulfide nanoparticles at different pump powers," J. Appl. Spectrosc. 71, 83-88 (2004).

15. V. G. Savitski, A. M. Malyarevich, K. V. Yumashev, V. L. Kalashnikov, B. D. Sinclair, H. Raaben, and A. A. Zhilin, "Experiment and modeling of a diode-pumped 1.3- $\mu \mathrm{m}$ $\mathrm{Nd}: \mathrm{YVO}_{4}$ laser passively $Q$-switched with PbS-doped glass," Appl. Phys. B 79, 315-319 (2004).

16. I. Kang and F. W. Wise, "Electronic structure and optical properties of $\mathrm{PbS}$ and PbSe quantum dots," J. Opt. Soc. Am. B 14, 1632-1646 (1997).

17. A. Declemey and C. Rulliere, "Totally automated picosecond transient absorption spectrometer using two photodiode arrays as detectors," Rev. Sci. Instrum. 57, 2733-2738 (1986).

18. J. L. Machol, F. W. Wise, R. C. Patel, and D. D. Tanner, "Vibronic quantum beats in $\mathrm{PbS}$ microcrystallites," Phys. Rev. B 48, 2819-2822 (1993).

19. M. M. Sheik-bahae, A. A. Said, T.-H. Wei, D. J. Hagan, and E. W. Van Stryland, "Sensitive measurements of optical nonlinearities using a single beam," IEEE J. Quantum Electron. 26, 760-769 (1990). 